\title{
MATRIX COMPARISON METHOD OF STATOR WINDINGS OF INDUCTION GENERATORS BY HARMONIC COMPOSITION OF MAGNETOMOTIVE FORCE
}

\author{
Nikolay Bogatyrev, Alexey Kramer, Vladimir Bogdan, Dmitrij Semernin \\ Kuban State Agrarian University named after I.T. Trubilin, Russia \\ bogatyrevn@yandex.ru,a-kram@mail.ru, alina48av@mail.ru,y007ta@yandex.ru
}

\begin{abstract}
At agricultural enterprises, squirrel-cage induction generators (IG), for which batch asynchronous motors (AM) can be used, are used as low-power generators of electric energy from renewable and traditional sources. The most difficult and technologically time-consuming part of $\mathrm{AM}$ is the stator winding. Its electromagnetic properties, manufacturability and cost determine the basic parameters of the machine - energy, economic efficiency, operational reliability and service life. Therefore, the choice of rational winding diagrams, research and optimization of their electromagnetic parameters for different connection diagrams of IG is a critical stage in the development and production of AM and IG. Induction generators use capacitances for selfexcitation and the higher harmonics in the EMF of the generator cause additional high frequency currents in these capacitances. The scheme analysis principles of three-phase stator windings of IG are discussed in the paper. It is indicated that the matrix analysis method of windings on their structural matrices may be possible. The paper describes the formalization technique using the Mathcad procedure for constructing and analyzing the magnetomotive forces (MMF) polygons. The MMF of windings and their harmonic composition up to 80 harmonics have been compared. It was shown that such indicators of the harmonic composition of MMF as: relative higher harmonic content, the harmonic coefficient quantitative values, along with the differential scattering coefficient, allow to compare the winding quality. This allows the determination and optimization of electromagnetic parameters of the proposed winding diagrams to minimize the higher harmonics content, depending on the coil performance and evaluate their effectiveness and compare different options for the winding diagrams. The study results suggest the future parameters of the induction machine and the possibility to use in induction generator mode at the design phase of the stator winding.
\end{abstract}

Keywords: induction generator, MMF polygon, higher harmonics, winding.

\section{Introduction}

The paper [1;2] examines features, development trends, methods of reducing motor losses, including asynchronous. Usually, there are the following types of basic losses according to the design of an asynchronous motor: electric, mechanical, electromagnetic and additional. The efforts to diminish losses are continuing in this area, and, ultimately, to increase the efficiency and energy efficiency class.

The developers of energy-efficient electric motors are solving the problem of increasing the efficiency by the following means [2]: using new grades of electrical-sheet steel for stator and rotor cores with lower specific losses and thickness of the core plates; reducing the air gap between the stator and rotor; reducing electromagnetic loads; increasing the cross-sectional area of the stator winding and the copper space factor; using copper to make short circuit rotor windings instead of aluminum; using high-quality bearings and changing the geometry of the external fan.

The characteristics of induction machines are affected by their parameters, mainly due to the schematic features of stator windings and design features of rotor windings. The well-known paper [3] considered the effect of higher harmonic fields on the stator and rotor windings. One of the techniques for construction of the Gerges diagram to determine the differential scattering is shown. The characteristics of different stator windings and their MMF analysis on the Gerges polygons are given.

Today's methods of forming stator windings enable by using methods to improve their diagrams to expand the search for ways to improve the energy efficiency of asynchronous motors [5-12].

\section{Materials and methods}

The quality of MMF of windings of any distributions is estimated by the minimum value of the differential scattering coefficient

$$
\tau_{\partial}=\left(\sum_{v=1}^{\infty} F_{v m}^{2} / F_{1 m}^{2}\right)-1,
$$


where $F_{1 m}, F_{v m}$ - fundamental $p$ and $v$ harmonic amplitudes of the stator winding MMF.

To exclude the calculation of the amplitudes of even a limited set of harmonics, the value of the differential scattering coefficient is calculated from the Gerges diagram.

When representing the MMF for three-phase winding as

$$
F_{x} e^{j\left(\omega t+\varphi_{x}\right)}=\int_{0}^{x} A_{x} e^{j\left(\omega t+\varphi_{x}\right)} d x=e^{j \omega t} \int_{0}^{x} A_{x} e^{j \varphi_{x}} d x
$$

closed figure of slot vectors

$$
A_{x} e^{j \varphi_{x}}=I_{1 m} N_{x} e^{j \varphi_{x}},
$$

where $\varphi_{x}$ - maximum current phase $I_{1 m}$ in $N$ efficient coil conductors, located at the slot with $x$ coordinate, and the Gerges diagram.

For the fundamental harmonic of the winding MMF with $N=2 m w / Z$ conductors in the slot, the Gerges diagram is a circle with a radius of:

$$
R_{P}=F_{1 m}=\frac{m I_{1 m} w k_{o b}}{\pi p}=\frac{Z N I_{1 m} k_{o b}}{2 \pi p} .
$$

By taking $I_{1 m} N=1, R_{P}=Z k_{o b} / 2 \pi p$ and the stator winding differential scattering coefficient

$$
\tau_{\partial}=\left(R_{\partial}^{2} / R_{p}^{2}\right)-1,
$$

where $R_{\partial}^{2}$ - polar moment of inertia of the slot points or spot tops of the Gerges diagram.
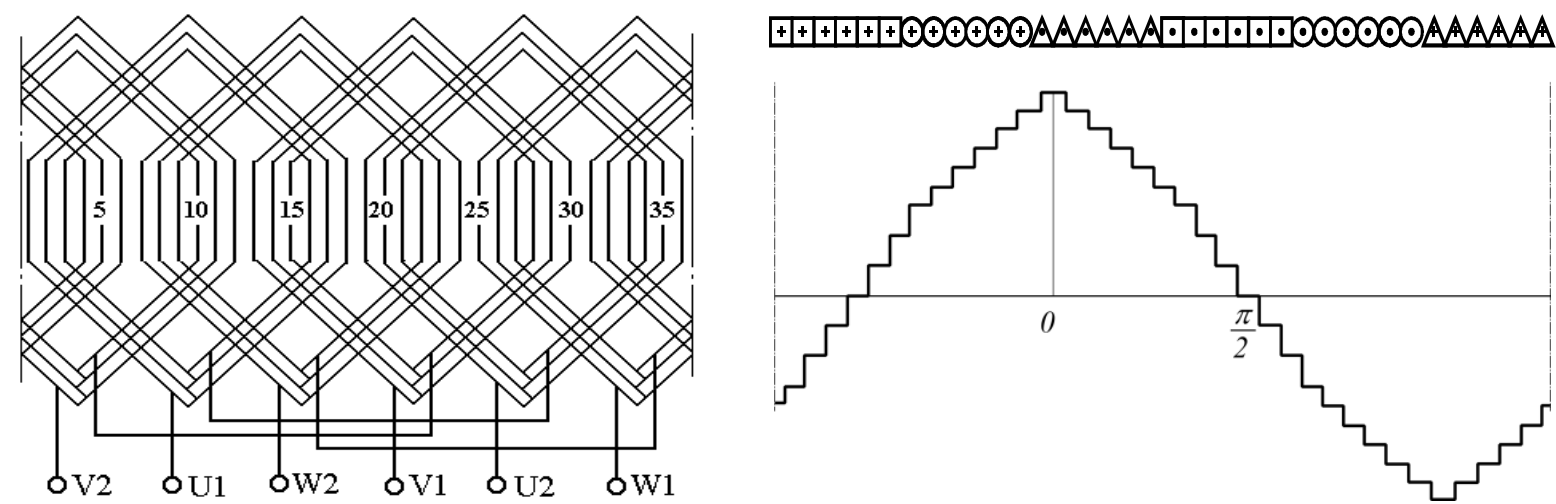

Fig. 1. Schematic diagram of a single-layer winding of the maximum distributions, its detailed diagram and MMF graph (1-36 number of stator slots)

For the analysis [12], we use the technique, taking as a basis the detailed winding diagram in the form of a table of Mathcad program, Figure 1, where a, b, c with the corresponding signs shows the detailed diagram of Table 1.

Table 1

The winding layout with the maximum distributions in Mathcad:a, $b, c$ with corresponding signs shows a detailed diagram in Figure 1

\begin{tabular}{|c|c|c|c|c|c|c|c|c|c|c|c|c|c|c|c|c|c|c|c|c|c|c|c|c|c|c|c|c|c|c|c|c|c|}
\hline & & & & & & & & & & & & & & & & & & & & & & & & & & & & & & & & & \\
\hline & 1 & 1 & 1 & 1 & 1 & 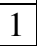 & 0 & & 0 & 0 & 7 & 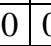 & 0 & 0 & & 0 & & & -1 & -1 & $1-$ & -1 & 0 & & 0 & & $\Omega$ & & & & & & \\
\hline & 0 & 0 & & 0 & & & & & 0 & & & & & 1 & & 1 & & & 0 & $\Omega$ & 4 & & & & & & & & & & & & \\
\hline & 0 & 0 & & 0 & 0 & & & & & & & & & 00 & & 0 & & & & & & & 1 & & & & & & & & & & \\
\hline
\end{tabular}

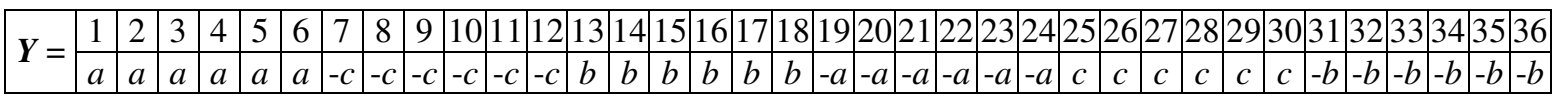

The structural matrix of the winding is as follows (1-36 number of stator slots)

Table 2 
Coordinates of the center of the MMF polygon are defined as

$$
X_{0} Y_{0}=\frac{|A\|M\| C \| P|^{T}}{Z}
$$

where $\mathrm{Z}$ - number of winding slots;

$|A|$ - unit vector;

$|M|$ - summation matrix;

$|C|$ - structure matrix of winding;

$|P|^{T}$ - transposed matrix of the direction of phase axes.

After centering, we have a MMF graph spread to the winding slots and a MMF polygon.

The estimated parameters of the winding:

- distribution factor $k_{o b}=0.95614$;

- fundamental harmonic radius of MMF $R=5.47829$;

- polar moment of inertia of the slot points $R_{p}^{2}=30.1667$;

- differential scattering coefficient $\tau_{d}=0.005163$.
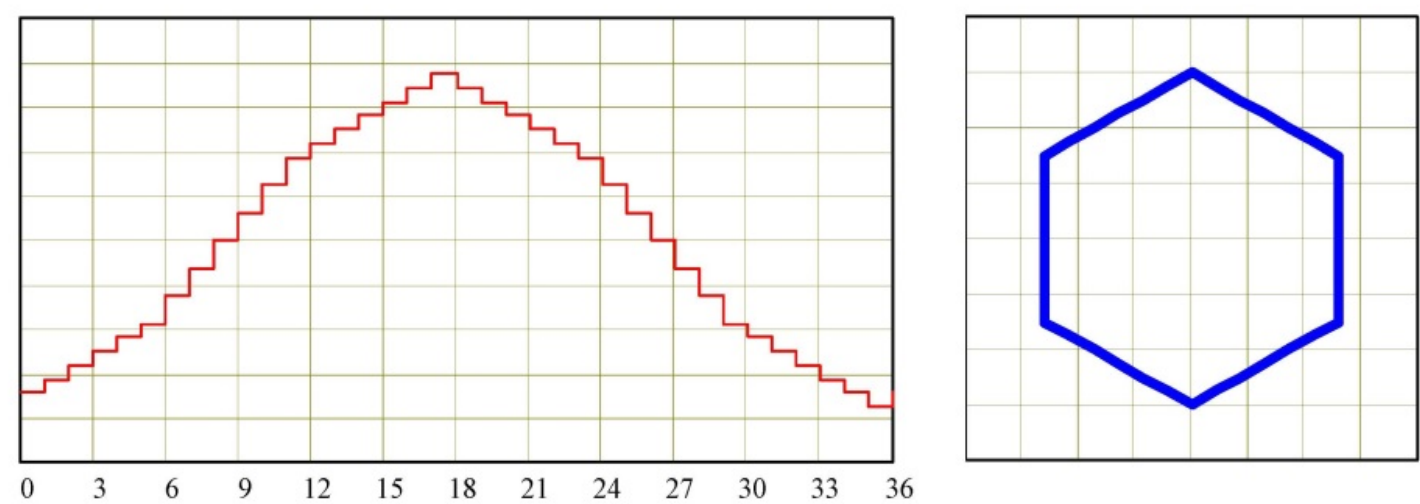

Fig. 2.Steps of the MMF curve in 36 grooves (left) and the polygon of the MMF winding of the maximum distribution (right)

The winding quality in addition to the differential scattering coefficient is also determined by the harmonic composition of MMF. The estimation of the harmonic composition of MMF from Gerges polygon has two disadvantages.

The first one is that the number of diagram slot points is limited by the number of slots. If we consider the stages of the MMF curve from $\mathrm{Z}$ slots as discretization points, according to Kotelnikov's theorem, the maximum frequency that we can calculate will be the $Z / 2$ frequency, that is, this is the frequency of the 18th harmonic for this winding.

The second one is that the differential scattering coefficient does not change, if there is a slot skewing, when the graph of MMF changes can be presented not by step, but by linear change of the MMF from slot to slot.

If we want to increase the maximum frequency of the harmonics found, the number of discretization points for this MMF curve should be increased in the interval between slots.

The authors defined the discretization, which is 4 times higher than the maximum number of a harmonic to be calculated. Then the harmonic analysis carried out from the expressions

- $\quad$ sine component -

$$
A_{n G}=\frac{1}{\pi} \sum_{k=1}^{Z} \sum_{n}^{N s} D_{k} \frac{\omega}{N S} \sin \left[n G \cdot \omega\left(k+\frac{n}{N S}\right)\right]
$$

- cosine component -

$$
B_{n G}=\frac{1}{\pi} \sum_{k=1}^{Z} \sum_{n}^{N s} D_{k} \frac{\omega}{N s} \cos \left[n G \cdot \omega\left(k+\frac{n}{N s}\right)\right],
$$


- harmonic amplitude -

$$
C_{n G}=\sqrt{A_{n G}^{2}+B_{n G}^{2}},
$$

where $N s-$ number of discretization points of one stage of the MMF graph,

$n G$ - number of harmonic to be defined,

$D_{k}$ - value of $\mathrm{k}$ stage of the MMF graph, provides the following result for harmonics above the first.

Total harmonic distortion of the MMF of this winding is

$$
k_{g}=\frac{\sqrt{\sum_{n G=2}^{80} C_{n G}^{2}}}{C_{n G=1}}=0.068428 .
$$

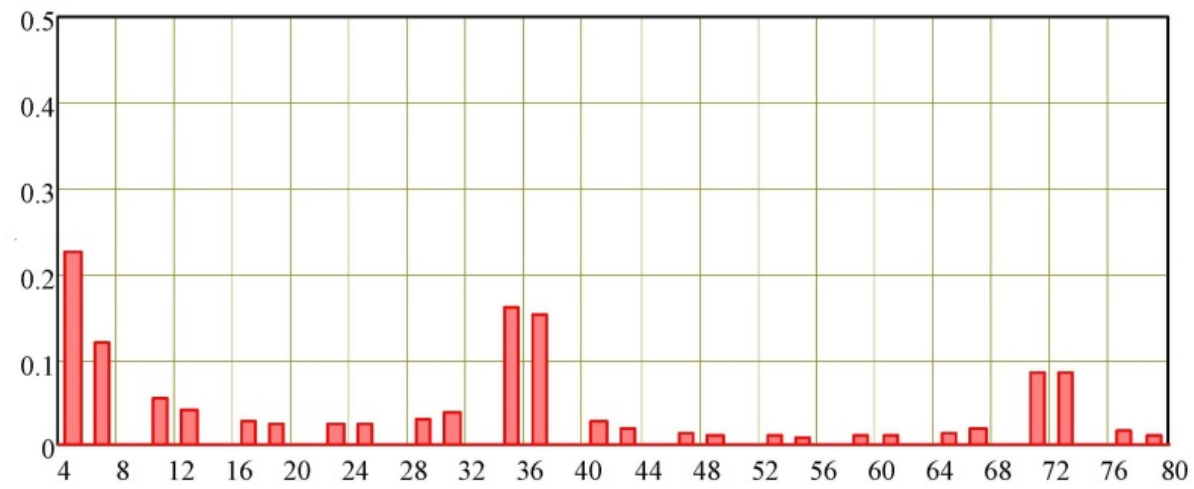

Fig. 3. Relative values of the MMF harmonics of the winding with maximum distributions

If we take into account the slot skewing, the MMF curve shape of the winding and the harmonic composition will change. The total harmonic distortion of MMF $k_{g}$ decreased by $20 \%$, although the Gerges MMF polygon remains unchanged.
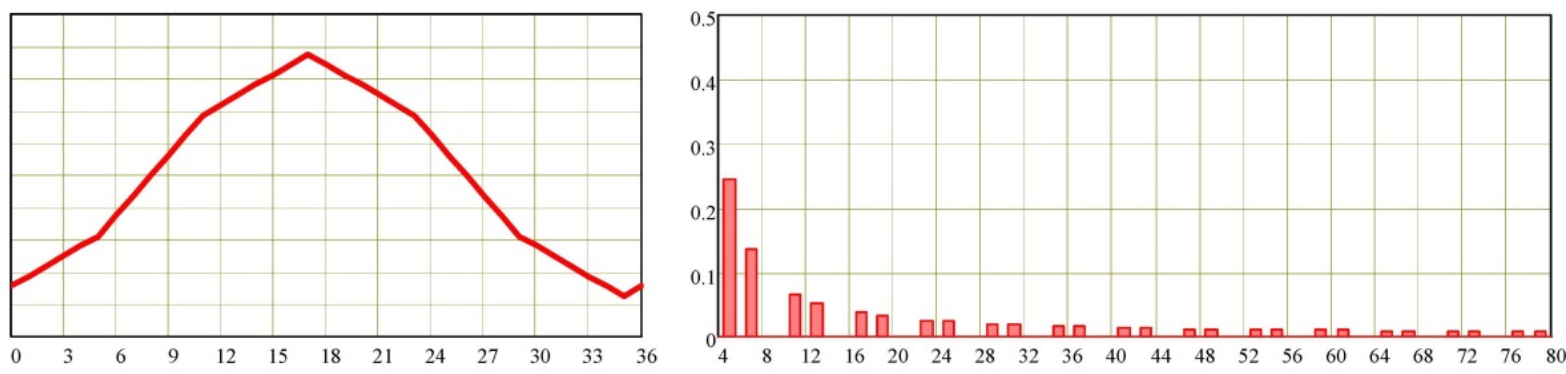

Fig. 4. MMF graph and the relative values of the MMF harmonics, if there is a slot skewing, $\boldsymbol{k}_{g}=\mathbf{0 . 0 5 4 9 5 8}$

\section{Results and discussion}

The analysis of windings for their use in different connection diagrams of IG was made according to the above technique.

The result of the calculation shows that the higher harmonics composition of the MMF curve is highly dependent on slot skewing that affects the stage equalization of the MMF curve. This is demonstrated by the data in Figures 5 and 6.

The difference in the values of higher harmonics decreases with the increase in the number of the stator winding slots. 


\section{Double-layer winding $Z=12$

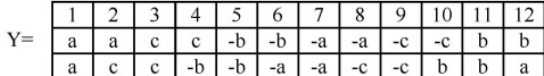

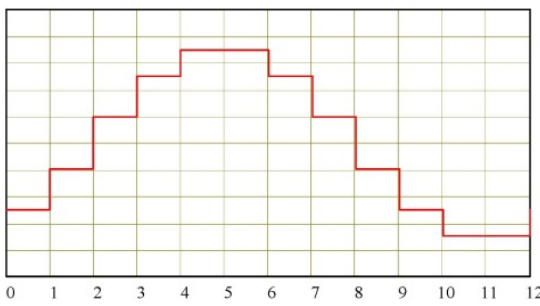
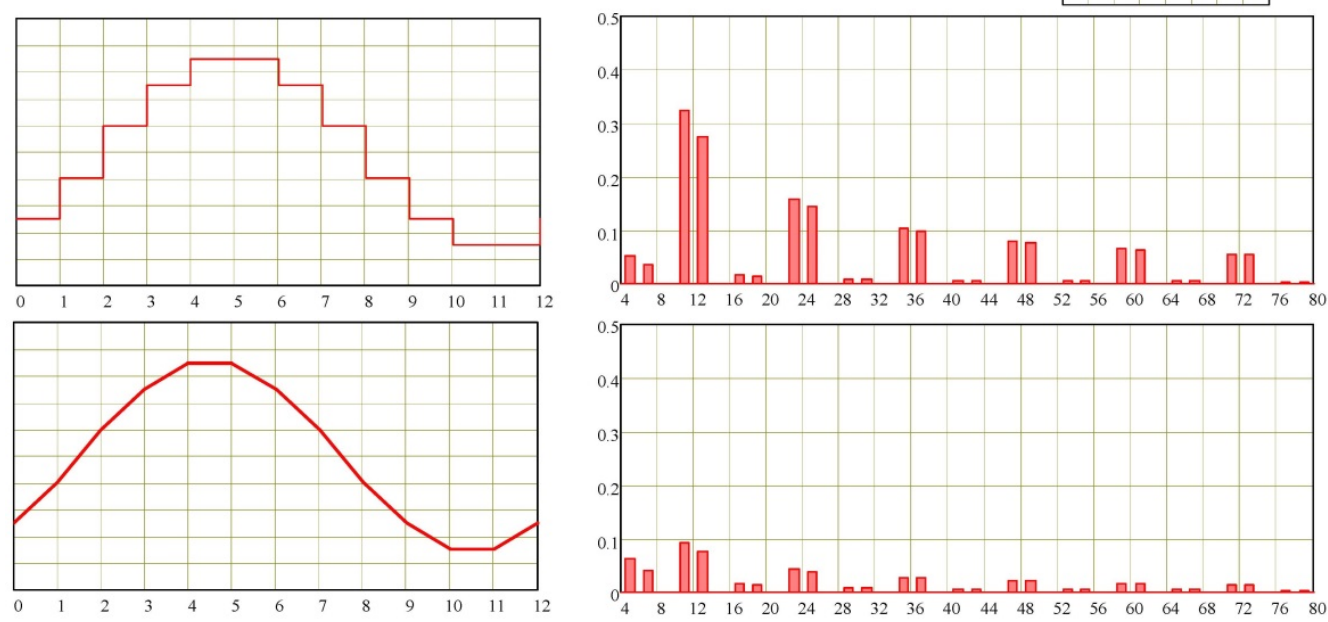

Fig. 5. Structural matrix of the winding and the polygon $\mathrm{MMF}$ ( top row), $k_{o b}=0.93301, R=3.562, R_{p}^{2}=13$, $\tau_{d}=0.023542$, without taking into account the bevel of the stator slots (middle row) $k_{g}=0.14773$, taking into account the bevel of the stator slots (bottom row) $k_{g}=0.045513$

In this case, the calculated total harmonic distortion has changed almost three times, when considering the slot skewing.

Double-layer winding $Z=24$
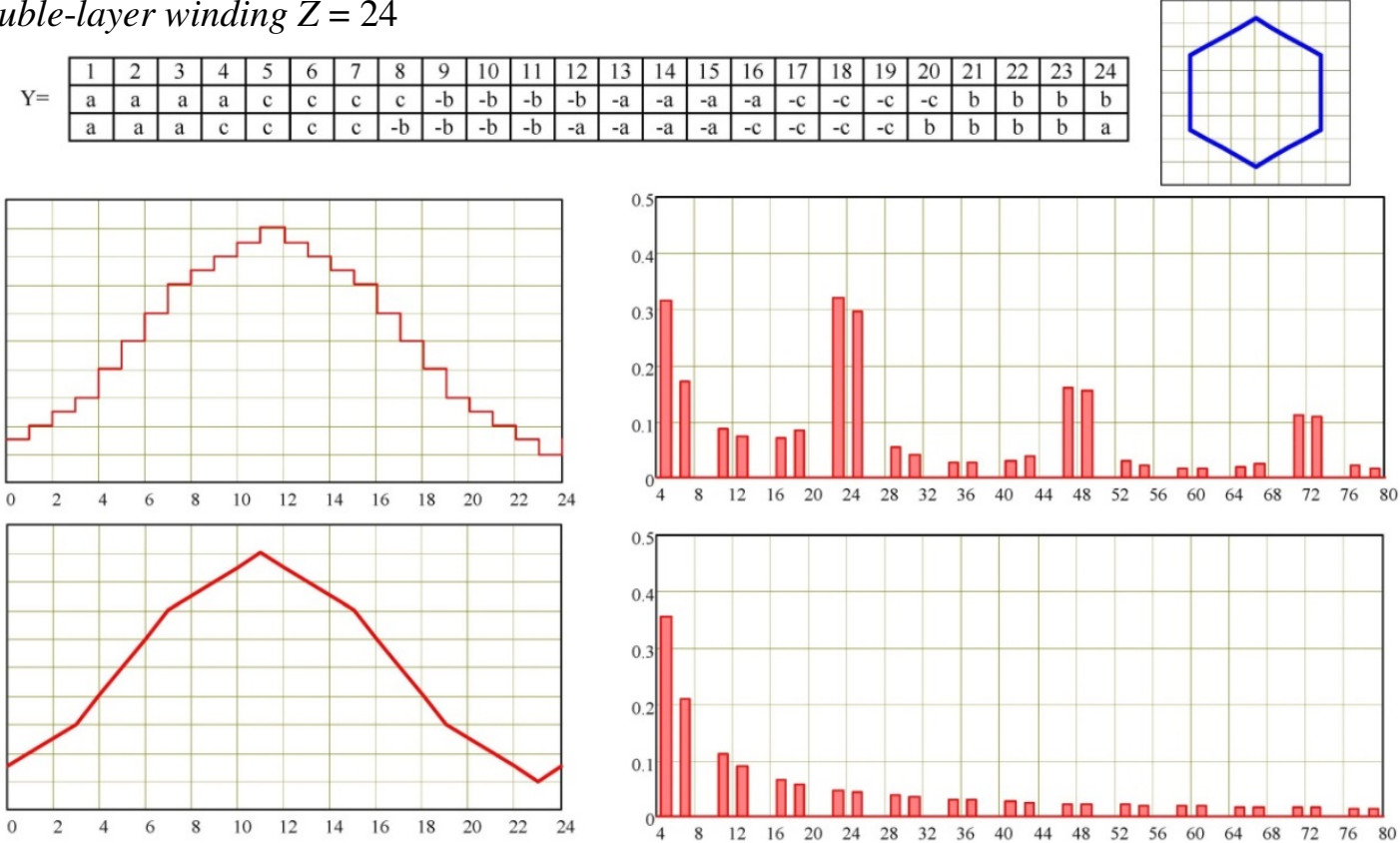

Fig. 6. Structural matrix of the winding and the polygon MMF ( top row) $k_{o b}=0.95776, R=3.658$, $R_{p}^{2}=13.5, \tau_{d}=0.008896$, without taking into account the bevel of the stator slots ( middle row) $k_{g}=0.089645$, taking into account the bevel of the stator slots (bottom row) $k_{g}=0.062662$

\section{Conclusions}

1. The assessment of the harmonic composition of the MDS by the Gerges polygon has two disadvantages. The first is that the number of slot points in the diagram is limited by the number of slots in the stator. If we count the steps of the MDS curve along the slots $\mathrm{Z}$ as sampling points, then according to Kotelnikov's theorem, the maximum frequency that we can calculate will be the frequency $\mathrm{Z} / 2$, that is, for this winding, it is the frequency of the 18 th harmonic. The second 
drawback is that the differential coefficient of dispersion does not change in the presence of the bevel grooves, when you can submit a schedule change of the MDS is not stepped but linear change of MDS from groove to groove.

2. Research has shown that depending on the number of stator slots, our calculation method reveals 10-15\% more harmonics than the known methods (up to 80 harmonics).

\section{References}

[1] Shumov Yu.N., Safonov A.S. Energy-Saving Electrical Machines (a Review of Foreign Developments), Electricity 2015, No. 4. pp. 45-57.

[2] Shumov Yu.N., Safonov A.S. Energy-Efficient Induction Motors with a Pressure\&Cast Copper Rotor Winding (a Review of Foreign Publications) Electricity 2014, No.8. Pages 56-61.

[3] Geller B., Gamata V. Higher harmonics in induction machines / Transl. from English. - M.: Energy, 1981. $-352 \mathrm{p}$.

[4] Zherve G.K. Windings of electrical machines L.: Energy, 1989 - 400 p.

[5] Bogatyrev N.I. Stator windings diagram, parameters and characteristics of alternating current machines: Monograph / N.I. Bogatyrev, V.N. Vanurin, O.V. Vronsky - Krasnodar, 2007. - 301 p.

[6] Makarov L.N. Improvement of serial induction machines in conditions of mass production, Electricity, 2005, No.7. pp. 62-69.

[7] Popov V. I. Matrix analysis of windings diagrams of combined electrical machines, Electricity, 1984, No.11. pp. 36-43.

[8] Popov V. I. Determination and optimization of electromagnetic parameters of three-phase fractional windings on MMF polygons, Electricity, 1997, No. 9, pp. 53-55.

[9] Popov V.I. Three-phase windings of RA series induction machines: systematization of diagrams and optimization of parameters, Electricity, 2001, No.6, pp. 22-32.

[10] Popov V.I. Harmonic analysis of MMF of three-phase fractional symmetric windings of electrical machines - Elektrotekhnika, 2008, No.5, pp. 61-67.

[11] Popov V.I. Improving the efficiency of three-phase single-layer windings of electrical machines, Electricity, 2009, No.1, pp. 63-69.

[12] Smirnov A.Yu. Matrix synthesis and analysis of MMF polygons of three-phase windings of alternating current machines, Electricity, 2009 No.5, pp. 42-45.

[13] Patent RU2680152 H02K 17/14 Autonomous induction generator with 12/10 pole-changing twolayer stator winding, Bogatyrev N.V., Vanurin V.N., Barakin N.S., Shablin V.E. Published: 18.02.2019 Bulletin No. 5. -9 p. 